\title{
Association of CT Perfusion Parameters with Hemorrhagic Transformation in Acute Ischemic Stroke
}

\author{
A.R. Jain, M. Jain, A.R. Kanthala, D. Damania, L.G. Stead, H.Z. Wang, and B.S. Jahromi
}

\begin{abstract}
BACKGROUND AND PURPOSE: Prediction of hemorrhagic transformation in acute ischemic stroke could help determine treatment and prognostication. With increasing numbers of patients with acute ischemic stroke undergoing multimodal CT imaging, we examined whether CT perfusion could predict hemorrhagic transformation in acute ischemic stroke.
\end{abstract}

MATERIALS AND METHODS: Patients with acute ischemic stroke who underwent CTP scanning within 12 hours of symptom onset were examined. Patients with and without hemorrhagic transformation were defined as cases and controls, respectively, and were matched as to IV rtPA administration and presentation NIHSS score $( \pm 2)$. Relative mean transit time, relative CBF, and relative CBV values were calculated from CTP maps and normalized to the contralateral side. Receiver operating characteristic analysis curves were created, and threshold values for significant CTP parameters were obtained to predict hemorrhagic transformation.

RESULTS: Of 83 patients with acute ischemic stroke, 16 developed hemorrhagic transformation (19.28\%). By matching, 38 controls were found for only 14 patients with hemorrhagic transformation. Among the matched patients with hemorrhagic transformation, 13 developed hemorrhagic infarction (6 hemorrhagic infarction 1 and 7 hemorrhagic infarction 2) and 1 developed parenchymal hematoma 2 . There was no significant difference between cases and controls with respect to age, sex, time to presentation from symptom onset, and comorbidities. Cases had significantly lower median rCBV ( $8 \%$ lower) compared with controls ( $11 \%$ higher) $(P=.009$; odds ratio, 1.14 for a $0.1-U$ decrease in $\mathrm{rCBV}$ ). There was no difference in median total volume of ischemia, rMTT, and rCBF among cases and controls. The area under the receiver operating characteristic was computed to be 0.83 (standard error, 0.08), with a cutoff point for rCBV of 1.09.

CONCLUSIONS: Of the examined CTP parameters, only lower rCBV was found to be significantly associated with a relatively higher chance of hemorrhagic transformation.

ABBREVIATIONS: AIS = acute ischemic stroke; $\mathrm{rCBF}=$ relative cerebral blood flow; $\mathrm{rCBV}=$ relative cerebral blood volume; $\mathrm{HI}=$ hemorrhagic infarction; $\mathrm{HT}=$ hemorrhagic transformation; IQR = interquartile range; $\mathrm{rMTT}=$ relative mean transit time; $\mathrm{ROC}=$ receiver operating characteristic analysis; $\mathrm{PH}=$ parenchymal hematoma

$\boldsymbol{S}^{\mathrm{tr} t a t}$ troke accounts for 1 in every 18 deaths in the United States, ${ }^{1}$ leaving nearly $30 \%$ of patients permanently disabled. ${ }^{2}$ Worldwide, stroke is a leading cause of disability and the fourth leading cause of mortality with 6.15 million deaths reported annually. ${ }^{3}$ In the United States alone, nearly 800,000 individuals have stroke each year, with a quarter of those being recurrent strokes. ${ }^{1,4}$

Many advances have been made in stroke prevention, imag-

Received May 18, 2012; accepted after revision December 16.

From the Departments of Neurosurgery (A.R.J., M.J., A.R.K., D.D., B.S.J.) and Imaging Sciences (H.Z.W.), University of Rochester Medical Center, Rochester, New York; and Departments of Emergency Medicine and Neurological Surgery (LG.S.), University of Florida College of Medicine, Gainesville, Florida.

Please address correspondence to Minal Jain, MBBS, MPH, Department of Neurosurgery, University of Rochester, 601 Elmwood Ave, Box 670, Rochester NY 14642; e-mail: minal_jain@urmc.rochester.edu

- Indicates open access to non-subscribers at www.ajnr.org

http://dx.doi.org/10.3174/ajnr.A3502 ing, and treatment during the past 20 years. The advent of IV rtPA for AIS in 1995 has improved outcomes for patients presenting within 3 hours, ${ }^{5}$ and with ongoing research, the time window for intravenous thrombolysis has been expanded. ${ }^{6-8}$ However, the use of IV rtPA at $>3$ hours also carries increased risk of hemorrhagic transformation, ${ }^{9}$ which remains a serious complication of ischemic stroke management and occurs in $4 \%-22 \%$ of patients with stroke. ${ }^{5,10,11}$ HT can be asymptomatic or symptomatic in 3\%-25\% of cases with resulting worsened outcome. ${ }^{12}$ Ongoing effort to find predictors of HT has yielded various factors, including the etiology of stroke, extent of infarction on initial CT, increasing stroke severity (NIHSS score), increasing age, congestive heart failure, high glucose levels, and blood pressure on presentation. ${ }^{11,13-17}$ Serum markers such as matrix metalloproteinase- 9 levels ${ }^{18}$; imaging modalities such as $\mathrm{CT},{ }^{19}$ SPECT, ${ }^{20}$ MR imaging, ${ }^{21}$ diffusion- and perfusion-weighted 

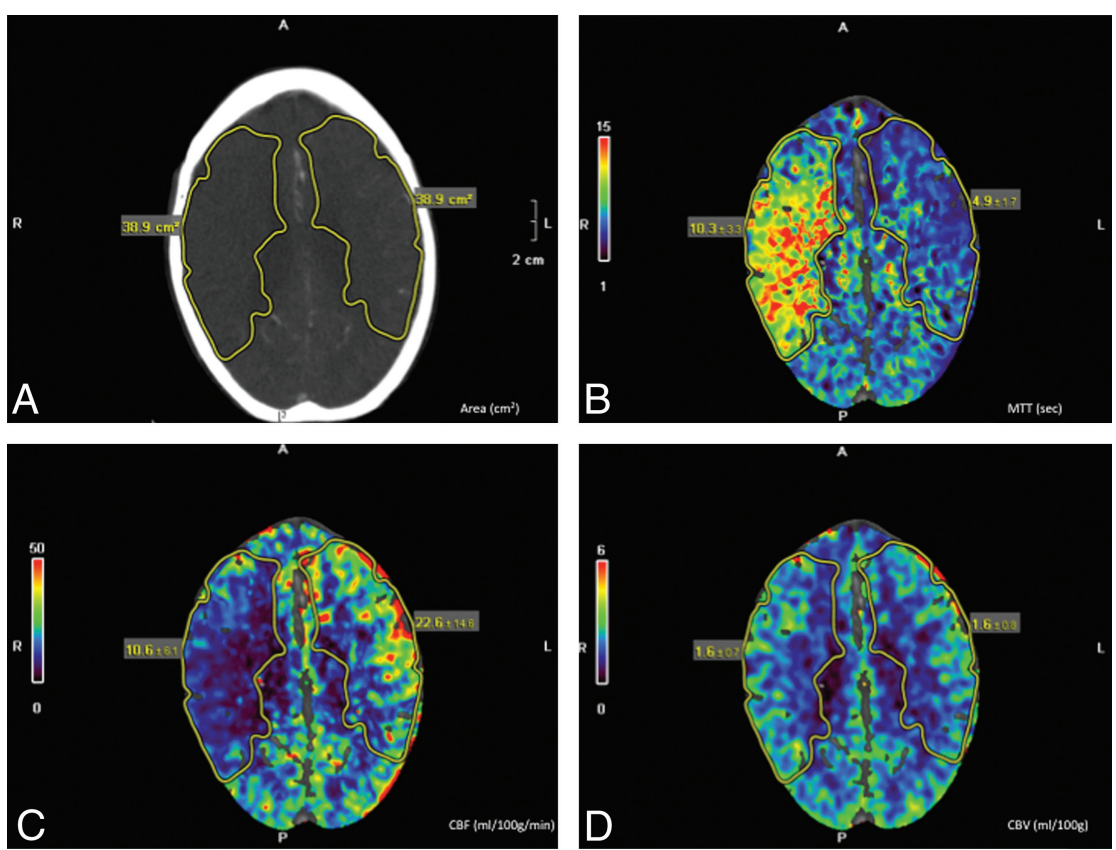

FIG 1. CTP maps. $A$, volume at risk of ischemia (volume computed from the area and thickness of each section [5 mm]). B, MTT. C, CBF. D, CBV. CTP images of a patient with AIS with right MCA occlusion. $\mathrm{rMTT}, \mathrm{rCBF}$, and $\mathrm{rCBV}$ are computed by normalizing $\mathrm{MTT}, \mathrm{CBF}$, and $\mathrm{CBV}$ of the ischemic area to the contralateral normal side. The outlined region of interest shows increased MTT, decreased $\mathrm{CBF}$, and preserved $\mathrm{CBV}$ in each respective panel.

\section{Table 1: Characteristics of study cohort}

\begin{tabular}{lc}
\multicolumn{1}{c}{ Characteristics } & $\begin{array}{c}\text { Total Patients } \\
(\boldsymbol{n}=\mathbf{8 3})\end{array}$ \\
\hline Female (No.) (\%) & $37(44.6 \%)$ \\
Age (yr) (median) (IQR) & $72(61-80)$ \\
Time (min) to arrival (median) (IQR) & $124(64-314)$ \\
Stroke severity on arrival (NIHSS) & $7(3-15)$ \\
$\quad$ (median) (IQR) & \\
Medical history & \\
$\quad$ Hypertension & $77.8 \%$ \\
$\quad$ Diabetes & $26.8 \%$ \\
$\quad$ Dyslipidemia & $52.4 \%$ \\
$\quad$ Stroke/TIA & $34.9 \%$ \\
$\quad$ Coronary artery disease & $24.4 \%$ \\
Medication history & \\
$\quad$ Antiplatelets & $45.0 \%$ \\
$\quad$ Anticoagulants & $10.1 \%$ \\
Thrombolysis done & $27.7 \%$ \\
Functional outcome (mRS) at discharge & $3(1-5)$ \\
$\quad$ (median) (IQR) & \\
Duration (days) of hospital stay (median) (IQR) & $5(3-8)$ \\
\hline
\end{tabular}

MR imaging $^{22}$; and blood-brain barrier permeability measures on $\mathrm{CTP}^{23}$ are also under investigation to formulate predictive models for HT.

In this study, we sought to investigate the hypothesis that there are inherent characteristics of ischemic brain tissue measureable by CTP that might predict hemorrhagic transformation. We evaluated the value of CBV, CBF, MTT, and volume of affected brain tissue toward prediction of HT in ischemic stroke.

\section{MATERIALS AND METHODS}

This was an institutional review board-approved study conducted at a single academic stroke center receiving $>700$ patients with ischemic stroke/TIA annually. The study extended from July 2009 to February 2010, and a cohort of all adult patients presenting with symptoms consistent with stroke/TIA within 12 hours of onset who underwent emergent CTP imaging was selected. At our institution, all patients with stroke presenting within 12 hours of symptom onset undergo CTP imaging. Patients with contraindications to CTP (ie, anaphylactic reaction to contrast, patients with known poor kidney function) were excluded. In addition, patients with a final diagnosis of TIA and those with infratentorial/bilateral/lacunar strokes were excluded. In this study, only patients with a discharge diagnosis of AIS were included in the final cohort.

The medical records were reviewed to obtain age, sex, medical history (hypertension, diabetes, dyslipidemia, coronary artery disease, TIA, stroke), smoking history, medication use, signs and symptoms on arrival, stroke severity at presentation (NIHSS score), use of IV rtPA and endovascular therapy, in-hospital outcome, and functional status on discharge (measured by the mRS). Follow-up noncontrast CT or MR imaging or both were reviewed to document the development of hemorrhagic transformation. "HT" was defined according to the European Cooperative Acute Stroke Study on NCCT as an area of increased attenuation within an area of low attenuation in a typical vascular distribution. ${ }^{24}$ On MR imaging, HT was identified by the presence of blood-product signal characteristics on T1, T2, and gradient-echo sequences. "Hemorrhagic transformation" was defined as per the European Cooperative Acute Stroke Study I and II trials and was classified as $\mathrm{HI}$ and $\mathrm{PH} .{ }^{15,24,25}$ This was further subdivided into HI 1 and HI 2 and PH 1 and PH 2. "HI 1" was defined as small petechiae along the peripheral margins of infarct. Confluent petechiae within the infarcted region but without mass effect were classified as $\mathrm{HI} 2$. "PH 1" was defined as hematoma with a mass effect of $<30 \%$ of the infarcted volume, and a $\mathrm{PH}$ with a mass effect of $>30 \%$ of infarct volume was classified as PH 2.

The CTP imaging studies of every research patient were analyzed. CTP images were processed by using Vitrea software (Vital Images, Minnetonka, Minnesota) by using a delay-insensitive algorithm (see "Imaging Protocol" below for further details). A freehand region of interest was used to outline the total brain tissue at risk of ischemia as defined by increased MTT and corresponding decreased CBF. A mirrored region of interest was automatically generated by the Vitrea software for measurement of $\mathrm{CBF}, \mathrm{CBV}$, and MTT within the contralateral normal brain and was used to normalize CBF, CBV, and MTT values derived for the affected hemisphere. This process is shown in Fig 1. Of note, we considered further subanalyses by breaking down each perfusion parameter measurement into 2 further values (for penumbral and 
Table 2: Comparison of cases and controls (matched on IV rtPA and NIHSS \pm 2 )

\begin{tabular}{lccc}
\hline \multicolumn{1}{c}{ Characteristics } & Cases $(\boldsymbol{n}=\mathbf{1 4})$ & Controls $(\boldsymbol{n}=\mathbf{3 8})$ & $\boldsymbol{P}$ Value \\
\hline Age (yr) (median) (IQR) & $71(61-77)$ & $75(65-84)$ & .15 \\
Female (No.) (\%) & $4(28.6 \%)$ & $19(50.0 \%)$ & .22 \\
Time (min) to arrival (median) (IQR) & $127.5(25-391)$ & $134(67-547)$ & .23 \\
Arrival systolic BP (mm Hg) (median) (IQR) & $146.5(136-171)$ & $153(134-162)$ & .87 \\
Arrival diastolic BP (mm Hg) (median) (IQR) & $81.5(75-98)$ & $83(66-102)$ & .74 \\
Arrival glucose level (mg/dL) (median) (IQR) & $123.5(107-141)$ & $120.5(104-142)$ & .85 \\
Arrival INR (median) (IQR) & $1(1-1.1)$ & $1.1(1-1.2)$ & .15 \\
Medical history & & & \\
$\quad$ Hypertension & $85.7 \%$ & $75.0 \%$ & .71 \\
Diabetes & $21.4 \%$ & $29.7 \%$ & .73 \\
$\quad$ Dyslipidemia & $57.1 \%$ & $54.1 \%$ & .84 \\
$\quad$ Stroke/TIA & $35.7 \%$ & $29.7 \%$ & .68 \\
$\quad$ Coronary artery disease & $42.9 \%$ & $16.2 \%$ & .05 \\
Antiplatelet use & $50 \%$ & $38.9 \%$ & .48 \\
Smoking & $43.2 \%$ & $27.0 \%$ & .20 \\
\hline
\end{tabular}

Note:-BP indicates blood pressure; INR, International Normalized Ratio.

Table 3: Comparison of CTP parameters for matched cases and controls after excluding patients having undergone endovascular therapy ${ }^{a}$

\begin{tabular}{cccc}
\hline Parameter & Cases $(\boldsymbol{n}=\mathbf{7})$ & Controls $(\boldsymbol{n}=\mathbf{2 4})$ & $\boldsymbol{P}$ Value \\
\hline MTTv (median) (IQR) & $209.6(48.5-345.6)$ & $147.6(78.53-235.8)$ & .5548 \\
Mean \pm SD & $194.91(136.98)$ & $161.59(115.19)$ & \\
rMTT (median) (IQR) & $1.83(1.42-2.23)$ & $1.80(1.50-2.16)$ & .8873 \\
Mean \pm SD & $1.87(0.43)$ & $2.20(1.70)$ & \\
rCBF (median) (IQR) & $0.54(0.40-0.63)$ & $0.58(0.46-0.70)$ & .5079 \\
Mean \pm SD & $0.52(0.14)$ & $0.58(0.15)$ & \\
rCBV (median) (IQR) & $0.92(0.83-1.07)$ & $1.11(1-1.29)$ & $.0093^{\text {b }}$ \\
Mean \pm SD & $0.93(0.13)$ & $1.19(0.36)$ & \\
\hline
\end{tabular}

Note:-MTTV indicates volume at risk of ischemia.

a Seven cases (accounting for 13 controls) and 1 additional control who received endovascular therapy were excluded. btatistically significant $(P<.05)$.

core infarct regions, based on the CBV). ${ }^{26}$ However, our sample size limited our ability to make multiple comparisons, and we, therefore, chose to proceed with measurements encompassing the entire ischemic region, as was done by previous authors. ${ }^{27}$

A matched case-control cohort was created. Any patient who had HT on follow-up imaging (CT or MR imaging) was considered a "case." Corresponding controls $(\leq 4)$ were selected for each of the cases by matching based on IV rtPA administration and presenting NIHSS score $( \pm 2)$. The matching was performed by using SAS (SAS Institute, Cary, North Carolina) (percentage match, greedy method).

Nonparametric analysis was performed to compare cases and controls by using the Wilcoxon rank sum test. For the initial analysis, all patients with endovascular therapy were excluded. CTP parameters were compared for cases and controls by using the Wilcoxon rank sum test, and $P$ values $<.05$ were considered significant. Analysis was then repeated, including endovascular patients as well, but excluding those with $\mathrm{SAH}$ or $\mathrm{PH}$ that might be interpreted as procedure-related (1 endovascular patient with $\mathrm{PH}$ was therefore excluded). Conditional logistic regression was used to analyze the effect of CTP parameters on the development of hemorrhagic transformation, and ROC curves were created. All analyses were performed in SAS 9.2/JMP 9 (SAS Institute).

\section{Imaging Protocol}

CTP imaging was performed on a 64-detector CT scanner (Brilliance; Philips Healthcare, Best, the Netherlands) following an initial noncontrast CT scan. This involved successive gantry rotations during IV administration of $50 \mathrm{~mL}$ of ioversol (Optiray 350; Mallinck- rodt, St. Louis, Missouri) at $5 \mathrm{~mL} / \mathrm{s}$ followed by a $40-\mathrm{mL}$ saline push. Coverage of $40 \mathrm{~mm}$ was obtained, with $8 \times 5 \mathrm{~mm}$ thick sections, $32 \times 1.25 \mathrm{~mm}$ collimation, and standard reconstruction. Acquisition parameters were $80 \mathrm{kV}$ (peak), $150 \mathrm{mAs}$; 2-second cycle time; 30 cycles; 0.4 -second rotation; and FOV, 230.

Postprocessing of images was performed by using Vitrea software, Version 3.0.1 (Vital Images). We used whole values derived from each region of interest rather than threshold values, because we have found that data derived from our commercial CTP software is more semiquantitative in nature and we feared that choosing a set uniform threshold across all patients would not account for differences in cardiac output, anatomy, and timing of injections among individual patients. Visually matched areas of increased MTT and decreased CBF were, therefore, outlined as ischemic areas, and all values were then reported as relative to the contralateral hemisphere, to account for potential interpatient variability noted above. The Vitrea software automatically chooses the selected arterial input function and venous output function (typically a proximal A2 vessel and a large dural sinus, respectively). These are verified by the technologist to be anatomically accurate and to result in correct time-to-peak maps of arterial and venous time-intensity curves. In rare cases in which there are discrepancies in the automatic arterial input function and venous output function locations, the arterial input function and venous output function are manually selected by the technologist. We additionally verified each arterial input function and venous output function for all patients included in the study.

\section{RESULTS}

\section{Characteristics of the Patient Population}

Eighty-three patients with stroke were enrolled in the study. The characteristics of the cohort are presented in Table 1. Of the total cohort, 16 (19.3\%) patients developed HT during their hospital stay. Of these, $37.5 \%$ had an early HT (HT within 36 hours of symptom onset). The median duration to the development of HT was 38.7 hours (IQR, 18.8-52.4 hours).

\section{Characteristics of Matched Cases and Controls}

After matching for IV rtPA administration and presenting NIHSS score $( \pm 2), 38$ controls were found for 14 cases (no controls were found for 2 patients with HT). There were no differences between cases and controls with respect to age, sex, vital parameters on presentation, history of disease or medication use, initial laboratory investigations, and smoking history (Table 2). Cases had a longer hospital stay (median, 8.5 days; IQR, 7-12 days) compared with controls (median, 5 days; IQR, 3-8 days; $P=.012$ ). There 


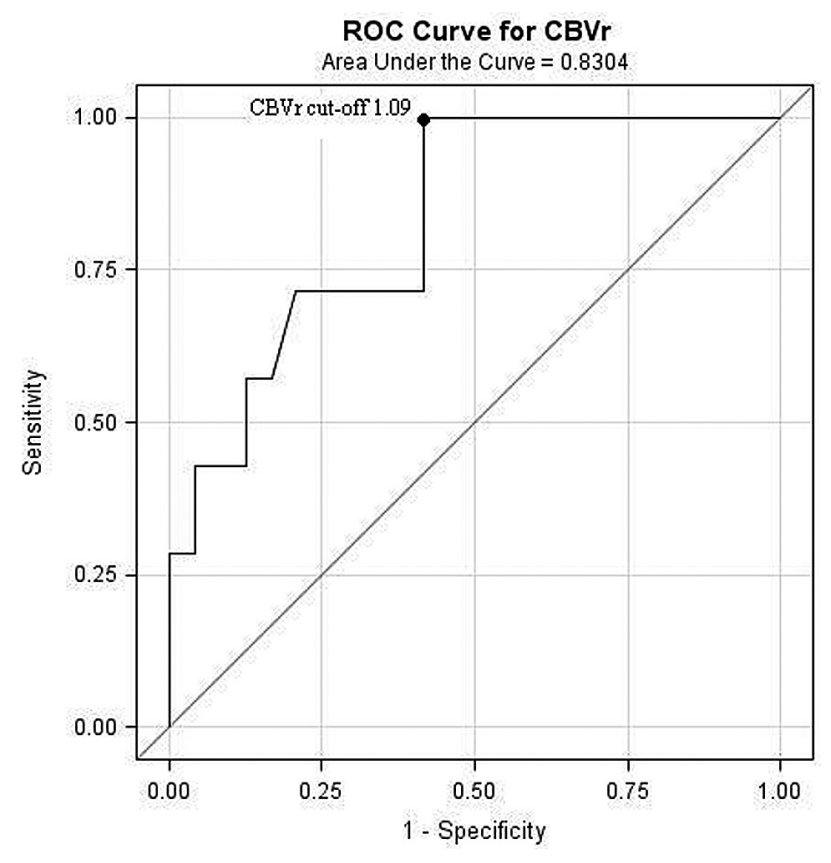

FIG 2. ROC curve of $\mathrm{rCBV}$ in patients with and without $\mathrm{HT}$, excluding all patients receiving endovascular therapy $(7$ cases [accounting for 13 controls] and 1 additional control who received endovascular therapy were excluded). $r$ CBV represents CBV of the ischemic area normalized to the contralateral side. The cutoff point indicates the threshold at which rCBV can predict HT with optimal sensitivity and specificity.

was a statistically nonsignificant difference between functional outcome assessed at discharge between cases (median mRS score, 5; IQR, 3-5) and controls (median mRS score, 3.5; IQR, 1-5; P = .11 ). Of the 14 cases, 13 had HI (7 HI 1 and $6 \mathrm{HI} 2$ ) and 1 case developed PH 2. Seven cases and 1 control were noted to have undergone endovascular therapy for acute ischemic stroke.

\section{Comparison of Cases and Controls}

All cases and controls who had undergone endovascular therapy were excluded ( 7 cases [accounting for 13 controls] and 1 additional control who received endovascular therapy were excluded). As seen in Table 3, these remnant cases had higher median total volume at risk of ischemia, longer median rMTT, and lower median $\mathrm{rCBF}$ compared with controls, though this did not reach statistical significance. Cases did have a significantly lower median rCBV compared with controls (median rCBV of cases, 0.92; IQR, 0.83-1.07 versus median rCBV of controls, 1.11; IQR, 1.0$1.29 ; P=.01$ ). For each $0.1-\mathrm{U}$ decrease in $\mathrm{rCBV}$, the odds of developing an HT increased by $14 \%$ (OR, 1.14; 95\% CI, 1.05-

Table 4: Comparison of CTP parameters for matched cases and controls ${ }^{a}$

\begin{tabular}{cccc}
\hline Parameter & Cases $(\boldsymbol{n}=13)$ & Controls $(\boldsymbol{n}=36)$ & $\boldsymbol{P}$ Value \\
\hline MTTv (median) (IQR) & $234.9(178.2-326.15)$ & $183.55(84.83-265.83)$ & .1968 \\
Mean \pm SD & $233.32(111.53)$ & $186.61(116.72)$ & \\
rMTT (median) (IQR) & $2.06(1.69-2.39)$ & $1.8(1.55-2.17)$ & .4020 \\
Mean \pm SD & $2.00(0.40)$ & $2.16(1.42)$ & \\
rCBF (median) (IQR) & $0.45(0.39-0.59)$ & $0.53(0.42-0.70)$ & .2572 \\
Mean \pm SD & $0.48(0.12)$ & $0.54(0.16)$ & \\
rCBV (median) (IQR) & $0.92(0.78-1.03)$ & $1.09(0.97-1.17)$ & $.0037^{\mathrm{b}}$ \\
Mean \pm SD & $0.92(0.17)$ & $1.13(0.33)$ & \\
\hline
\end{tabular}

Note:-MTTV indicates volume at risk of ischemia.

a Excluding cases with both endovascular therapy and SAH/PH $(n=1)$ and their corresponding controls $(n=2)$.

${ }^{b}$ Statistically significant $(p<.05)$.
1.25). The ROC curve for rCBV is shown in Fig 2. The area under the ROC curve was computed to be 0.83 (SE, 0.08 ; 95\% CI, $0.67-$ 0.99 ), with a cutoff point for rCBV of 1.09 . The sensitivity for this cutoff value was $100 \%$, and specificity was $58.3 \%$.

Considering that only SAH or PH can truly be interpreted as being procedure-related hemorrhage after endovascular therapy, we repeated the above analysis (excluding cases with both endovascular therapy and $\mathrm{SAH} / \mathrm{PH}[n=1]$ and their corresponding controls $[n=2])$. Repeat analysis depicted in Table 4 yielded similar results as before: Cases had higher volume at risk of ischemia, longer median rMTT, and lower median rCBF compared with controls. However, this again did not reach statistical significance. The median rCBV was significantly lower in cases compared with controls (median CBVr of cases, 0.92; IQR, 0.78-1.03 versus median rCBV of controls, 1.09; IQR, 0.97-1.17; $P=.004$ ). For each $0.1-\mathrm{U}$ decrease in $\mathrm{rCBV}$, the odds of developing an HT increased by $12 \%$ (OR, 1.12; 95\% CI, 1.05-1.19). The ROC curve for $\mathrm{rCBV}$ is shown in Fig 3. The area under the ROC curve was computed to be 0.78 (SE, 0.08 ; 95\% CI, 0.62-0.94), with a cutoff point for rCBV of 0.98 . The sensitivity for a cutoff value of $\mathrm{rCBV}$ was $76.92 \%$ with a specificity of $72.22 \%$.

\section{DISCUSSION}

The incidence of HT has been reported to vary between $2 \%$ and $44 \%$ of patients with ischemic stroke, ${ }^{5,11,24}$ and the $19.3 \%$ rate observed in our study falls within this range. Besides thrombolytic therapy, HT has been associated with age younger than 65 years, ${ }^{28}$ hyperglycemia, ${ }^{29}$ acute hypertension ${ }^{30}$ and blood pressure variability, ${ }^{31}$ low levels of coated platelets, ${ }^{32}$ and cardioembolic stroke subtype. ${ }^{33}$ As new avenues are explored to stretch the time window for beneficial therapy, ${ }^{34}$ there is an increasingly acute need to accurately predict the likelihood of HT in every patient eligible for intervention to judicially triage these patients to available therapies while reducing complications.

Prediction of HT by reduced pretherapeutic CBF, an inherent characteristic of the stroke itself, was first suggested by Ueda et $\mathrm{al}^{35}$ by using SPECT. A reduction in CBF to $<50 \%$ of normal was considered as the critical value for developing HT. ${ }^{36}$ Gupta et al ${ }^{37}$ in their study of 23 patients with symptomatic stroke or carotid stenosis concluded that mean ipsilateral $\mathrm{CBF}<13 \mathrm{~mL} / 100$ g per minute was the cutoff for developing HT. In our cohort, we found that $\mathrm{rCBV}$ rather than $\mathrm{rCBF}$ was the strongest predictor of HT. This is similar to reports of rCBV (rather than rCBF) being a stronger predictor of penumbra viability in patients with AIS, ${ }^{26}$ with similar ${ }^{38}$ or even lower ${ }^{39}$ values reported by authors for rCBV in patients with HT. In other words, indicators of penumbra viability may also indirectly predict HT, which would be expected to occur more frequently in infarcted core rather than salvageable penumbra regions. We found in our patients that a cutoff $\mathrm{rCBV}$ of at least 0.98 could predict development of HT in patients with AIS with $72 \%$ specificity.

The role of imaging in acute stroke is ever-expanding. ${ }^{40,41}$ Many modalities 


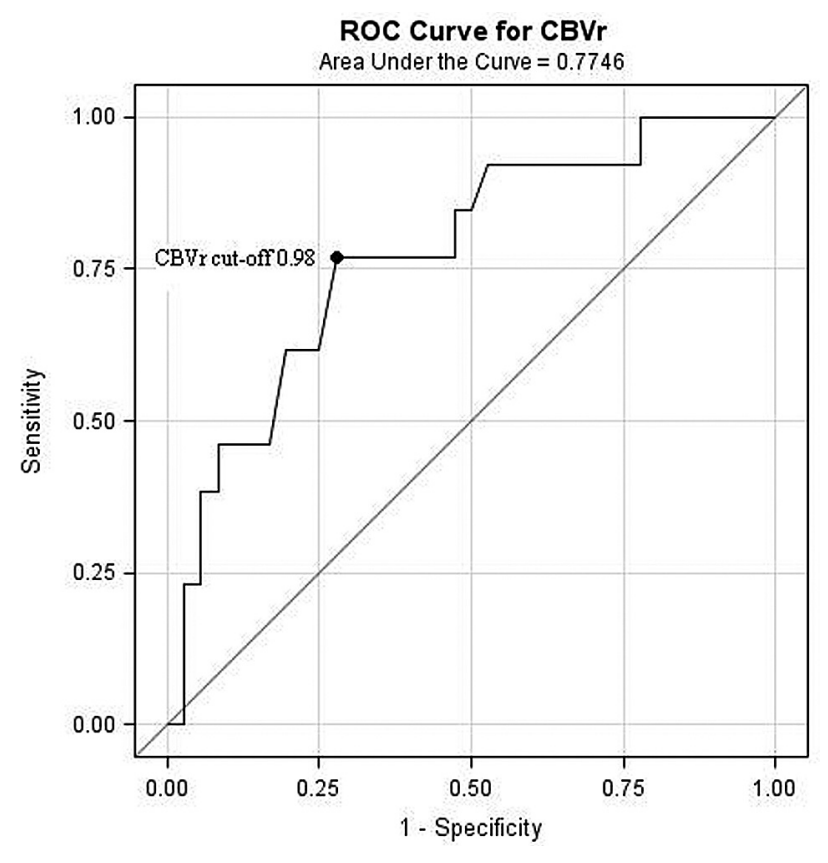

FIG 3. ROC curve of $\mathrm{rCBV}$ in patients with and without $\mathrm{HT}$ (excluding cases with both endovascular therapy and $\mathrm{PH} / \mathrm{SAH}[n=1]$ and their corresponding controls [ $n=2]$ ). rCBV represents $C B V$ of the ischemic area normalized to the contralateral side. The cutoff point indicates the threshold at which rCBV can predict HT with optimal sensitivity and specificity.

have shown promise in delineating both the extent of viable brain tissue within the area at ischemic risk as well as in predicting HT. The high risk of HT was associated in the past with an M1 MCA occlusion and lack of collateral flow evaluated by transcranial Doppler, SPECT, and clinical criteria (Toronto Embolic Scale). ${ }^{20}$ More recently, BBB permeability studies via MR and CTP imaging have been shown to have a high sensitivity for predicting symptomatic HT. ${ }^{23,42}$ The ROC for rCBV in our study suggests that CBV may also predict HT. The utility of this finding lies in the fact that CT imaging is already a part of the imaging protocol in a large number of tertiary care institutions. CTP as an add-on can be performed easily and quickly in an emergent setting, while providing information about tissue-level hemodynamics, extent of penumbra, and final infarct size. ${ }^{43}$ Should our findings be replicated on a larger scale, an additional utility would be prediction of HT, which could further guide emergent treatment decisions for patients with acute stroke.

\section{Limitations}

There are several limitations to this study. This was a retrospective study. Effects of stroke severity at presentation and IV rtPA administration were controlled for by matching; however, this reduced the final cohort size. No adjustments were made for specific anatomic locations of perfusion parameters and region-specific risk of HT. Our results represent only a single-institution experience and have not been validated and may, therefore, not be representative of community practice.

\section{CONCLUSIONS}

Our data suggest that CTP imaging and, in particular, low rCBV can predict HT in patients with AIS. Should this exploratory anal- ysis be validated in larger studies, initial multimodal CT imaging, including CTP, could be used to triage patients at high risk of HT to treatments less likely to further exacerbate their inherent risk of impending intracerebral hemorrhage.

\section{REFERENCES}

1. Lloyd-Jones D, Adams RJ, Brown TM, et al. Heart disease and stroke statistics: 2010 update - a report from the American Heart Association. Circulation 2010;121:e46-e215

2. Asplund K, Stegmayr B, Peltonen M. From the twentieth to the twenty-first century: a public health perspective on stroke. In: Ginsberg MD, Bogousslavsky J, eds. Cerebrovascular Disease Pathophysiology, Diagnosis, and Management. Vol 2. Malden, Massachusetts: Blackwell Science; 1998

3. World Health Organization. The top 10 causes of death: updated 2011. http://www.who.int/mediacentre/factsheets/fs310/en/index.html. Accessed March 12, 2012

4. Incidence and Prevalence: 2006 Chart Book on Cardiovascular and Lung Diseases. Bethesda, Maryland: National Institutes of Health, National Heart Lung and Blood institute; 2006

5. Tissue plasminogen activator for acute ischemic stroke: the National Institute of Neurological Disorders and Stroke rt-PA Stroke Study Group. N Engl J Med 1995;333:1581-87

6. Hacke W, Kaste M, Bluhmki E, et al. Thrombolysis with alteplase 3 to 4.5 hours after acute ischemic stroke. $N$ Engl $\mathrm{J} \mathrm{Med}$ 2008;359:1317-29

7. Wahlgren N, Ahmed N, Davalos A, et al. Thrombolysis with alteplase 3-4.5 $\mathrm{h}$ after acute ischaemic stroke (SITS-ISTR): an observational study. Lancet 2008;372:1303-09

8. Ahmed N, Wahlgren N, Grond M, et al. Implementation and outcome of thrombolysis with alteplase $3-4.5 \mathrm{~h}$ after an acute stroke: an updated analysis from SITS-ISTR. Lancet Neurol 2010;9:866-74

9. Lees KR, Bluhmki E, von Kummer R, et al. Time to treatment with intravenous alteplase and outcome in stroke: an updated pooled analysis of ECASS, ATLANTIS, NINDS, and EPITHET trials. Lancet 2010;375:1695-703

10. Jaillard A, Cornu C, Durieux A, et al. Hemorrhagic transformation in acute ischemic stroke: the MAST-E study-MAST-E Group. Stroke 1999;30:1326-32

11. Larrue V, von Kummer RR, Muller A, et al. Risk factors for severe hemorrhagic transformation in ischemic stroke patients treated with recombinant tissue plasminogen activator: a secondary analysis of the European-Australasian Acute Stroke Study (ECASS II). Stroke 2001;32:438-41

12. Kerenyi L, Kardos L, Szasz J, et al. Factors influencing hemorrhagic transformation in ischemic stroke: a clinicopathological comparison. Eur J Neurol 2006;13:1251-55

13. Kidwell CS, Saver JL, Carneado J, et al. Predictors of hemorrhagic transformation in patients receiving intra-arterial thrombolysis. Stroke 2002;33:717-24

14. Dzialowski I, Pexman JH, Barber PA, et al. Asymptomatic hemorrhage after thrombolysis may not be benign: prognosis by hemorrhage type in the Canadian alteplase for stroke effectiveness study registry. Stroke 2007;38:75-79

15. Fiorelli M, Bastianello S, von Kummer R, et al. Hemorrhagic transformation within 36 hours of a cerebral infarct: relationships with early clinical deterioration and 3-month outcome in the European Cooperative Acute Stroke Study I (ECASS I) cohort. Stroke 1999;30:2280-84

16. Larrue V, von Kummer R, del Zoppo G, et al. Hemorrhagic transformation in acute ischemic stroke: potential contributing factors in the European Cooperative Acute Stroke Study. Stroke 1997;28:957-60

17. Dowlatshahi D, O'Donnell M, Sharma M. Possible influence of stroke etiology on hemorrhagic transformation following thrombolysis in warfarin-treated patients. Arch Neurol 2010;67:1413, author reply 1414 
18. Castellanos M, Leira R, Serena J, et al. Plasma metalloproteinase-9 concentration predicts hemorrhagic transformation in acute ischemic stroke. Stroke 2003;34:40-46

19. Toni D, Fiorelli M, Bastianello S, et al. Hemorrhagic transformation of brain infarct: predictability in the first 5 hours from stroke onset and influence on clinical outcome. Neurology 1996;46:341-45

20. Alexandrov AV, Black SE, Ehrlich LE, et al. Predictors of hemorrhagic transformation occurring spontaneously and on anticoagulants in patients with acute ischemic stroke. Stroke 1997;28:1198-202

21. Knight RA, Barker PB, Fagan SC, et al. Prediction of impending hemorrhagic transformation in ischemic stroke using magnetic resonance imaging in rats. Stroke 1998;29:144-51

22. Tong DC, Adami A, Moseley ME, et al. Prediction of hemorrhagic transformation following acute stroke: role of diffusion- and perfusion-weighted magnetic resonance imaging. Arch Neurol 2001;58:587-93

23. Hom J, Dankbaar JW, Soares BP, et al. Blood-brain barrier permeability assessed by perfusion CT predicts symptomatic hemorrhagic transformation and malignant edema in acute ischemic stroke. AJNR Am J Neuroradiol 2011;32:41-48

24. Hacke W, Kaste M, Fieschi C, et al. Intravenous thrombolysis with recombinant tissue plasminogen activator for acute hemispheric stroke: the European Cooperative Acute Stroke Study (ECASS). JAMA 1995;274:1017-25

25. Hacke W, Kaste M, Fieschi C, et al. Randomised double-blind placebocontrolled trial of thrombolytic therapy with intravenous alteplase in acute ischaemic stroke (ECASS II): Second European-Australasian Acute Stroke Study Investigators. Lancet 1998;352:1245-51

26. Parsons MW, Pepper EM, Bateman GA, et al. Identification of the penumbra and infarct core on hyperacute noncontrast and perfusion CT. Neurology 2007;68:730-36

27. Aviv RI, d'Esterre CD, Murphy BD, et al. Hemorrhagic transformation of ischemic stroke: prediction with CT perfusion. Radiology 2009;250:867-77

28. Saver JL. Hemorrhage after thrombolytic therapy for stroke: the clinically relevant number needed to harm. Stroke 2007;38:2279-83

29. Broderick JP, Hagen T, Brott T, et al. Hyperglycemia and hemorrhagic transformation of cerebral infarcts. Stroke 1995;26:484-87

30. Bowes MP, Zivin JA, Thomas GR, et al. Acute hypertension, but not thrombolysis, increases the incidence and severity of hemorrhagic transformation following experimental stroke in rabbits. Exp Neurol 1996;141:40-46

31. Ko Y, Park JH, Yang MH, et al. The significance of blood pressure variability for the development of hemorrhagic transformation in acute ischemic stroke. Stroke 2010;41:2512-18

32. Prodan CI, Stoner JA, Cowan LD, et al. Lower coated-platelet levels are associated with early hemorrhagic transformation in patients with non-lacunar brain infarction. J Thromb Haemost 2010;8:1185-90

33. Okada Y, Yamaguchi T, Minematsu K, et al. Hemorrhagic transformation in cerebral embolism. Stroke 1989;20:598-603

34. Rowley HA. Extending the time window for thrombolysis: evidence from acute stroke trials. Neuroimaging Clin N Am 2005;15:575-87, x

35. Ueda T, Hatakeyama T, Kumon Y, et al. Evaluation of risk of hemorrhagic transformation in local intra-arterial thrombolysis in acute ischemic stroke by initial SPECT. Stroke 1994;25:298-303

36. Seki H, Yoshimoto T, Ogawa A, et al. Hemodynamics in hemorrhagic infarction: an experimental study. Stroke 1985;16:647-51

37. Gupta R, Yonas H, Gebel J, et al. Reduced pretreatment ipsilateral middle cerebral artery cerebral blood flow is predictive of symptomatic hemorrhage post-intra-arterial thrombolysis in patients with middle cerebral artery occlusion. Stroke 2006;37:2526-30

38. Souza LC, Payabvash S, Wang Y, et al. Admission CT perfusion is an independent predictor of hemorrhagic transformation in acute stroke with similar accuracy to DWI. Cerebrovasc Dis 2012;33:8-15

39. Lin K, Zink WE, Tsiouris AJ, et al. Risk assessment of hemorrhagic transformation of acute middle cerebral artery stroke using multimodal CT. J Neuroimaging 2012;22:160-66

40. Sims J, Schwamm LH. The evolving role of acute stroke imaging in intravenous thrombolytic therapy: patient selection and outcomes assessment. Neuroimaging Clin N Am 2005;15:421-40, xii

41. Schellinger PD. The evolving role of advanced MR imaging as a management tool for adult ischemic stroke: a Western-European perspective. Neuroimaging Clin N Am 2005;15:245-58, ix

42. Thornhill RE, Chen S, Rammo W, et al. Contrast-enhanced MR imaging in acute ischemic stroke: $\mathrm{T} 2{ }^{\star}$ measures of blood-brain barrier permeability and their relationship to $\mathrm{T} 1$ estimates and hemorrhagic transformation. AJNR Am J Neuroradiol 2010;31:1015-22

43. Wintermark M, Reichhart M, Thiran JP, et al. Prognostic accuracy of cerebral blood flow measurement by perfusion computed tomography, at the time of emergency room admission, in acute stroke patients. Ann Neurol 2002;51:417-32 